\title{
Somatic molecular analysis augments cytologic evaluation of pancreatic cyst fluids as a diagnostic tool
}

\author{
Ali Sakhdari ${ }^{1,5}$, Parnian Ahmadi Moghaddam ${ }^{1,4,6}$, Chi Young Ok ${ }^{1,5}$, Otto Walter ${ }^{1}$, \\ Keith Tomaszewicz ${ }^{1}$, Mandi-Lee Caporelli ${ }^{1}$, Xiuling Meng ${ }^{1}$, Jennifer LaFemina ${ }^{2}$, \\ Giles Whalen ${ }^{2}$, Edward Belkin ${ }^{3}$, Jaroslav Zivny ${ }^{3}$, Wahid Wassef ${ }^{3}$, Bruce A. Woda ${ }^{1}$, \\ Lloyd M. Hutchinson ${ }^{1}$ and Ediz F. Cosar ${ }^{1}$ \\ ${ }^{1}$ University of Massachusetts Medical School, Department of Pathology, Worcester, MA, USA \\ ${ }^{2}$ University of Massachusetts Medical School, Department of Surgery, Worcester, MA, USA \\ ${ }^{3}$ University of Massachusetts Medical School, Department of Medicine, Worcester, MA, USA \\ ${ }^{4}$ Massachusetts General Hospital, Department of Pathology, Boston, MA, USA \\ ${ }^{5}$ MD Anderson Cancer Center, Department of Hematopathology, Houston, TX, USA \\ ${ }^{6}$ University of Texas, Health Science Center, Department of Pathology, Houston, TX, USA \\ Correspondence to: Lloyd Hutchinson, email: Lloyd.Hutchinson@umassmemorial.org \\ Keywords: pancreatic cyst classification; non-diagnostic cytology; molecular next generation sequencing \\ Received: February 08, $2019 \quad$ Accepted: May 20, $2019 \quad$ Published: June 18, 2019 \\ Copyright: Sakhdari et al. This is an open-access article distributed under the terms of the Creative Commons Attribution License \\ 3.0 (CC BY 3.0), which permits unrestricted use, distribution, and reproduction in any medium, provided the original author and \\ source are credited.
}

\section{ABSTRACT}

Objective: Better tools are needed for early diagnosis and classification of pancreatic cystic lesions (PCL) to trigger intervention before neoplastic precursor lesions progress to adenocarcinoma. We evaluated the capacity of molecular analysis to improve the accuracy of cytologic diagnosis for PCL with an emphasis on nondiagnostic/negative specimens.

Design: In a span of 7 years, at a tertiary care hospital, 318 PCL endoscopic ultrasound-guided fine needle aspirations (EUS-FNA) were evaluated by cytologic examination and molecular analysis. Mucinous PCL were identified based on a clinical algorithm and 46 surgical resections were used to verify this approach. The mutation allele frequency (MAF) of commonly altered genes (BRAF, CDKN2A, CTNNB1, GNAS, RAS, PIK3CA, PTEN, SMAD4, TP53 and VHL) was evaluated for their ability to identify and grade mucinous PCL.

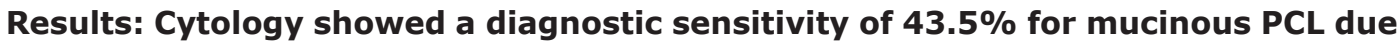
in part to the impact of non-diagnostic $(28.8 \%)$ and negative $(50.5 \%)$ specimens. Incorporating an algorithmic approach or molecular analysis markedly increased the accuracy of cytologic evaluation. Detection of mucinous PCL by molecular analysis was 93.3\% based on the detection of KRAS and/or GNAS gene mutations $(p=0.0001)$. Additional genes provided a marginal improvement in sensitivity but were associated with cyst type (e.g. VHL) and grade (e.g. SMAD4). In the surgical cohort, molecular analysis and the proposed algorithm showed comparable sensitivity $(88.9 \%$ vs. $100 \%$ ).

Conclusions: Incorporating somatic molecular analysis in the cytologic evaluation of EUS-FNA increases diagnostic accuracy for detection, classification and grading of PCL. This approach has the potential to improve patient management. 


\section{INTRODUCTION}

Recent advances in diagnostic imaging such as high-resolution ultrasonography (HR-US), computed tomography (CT), and magnetic resonance imaging (MRI) have increased detection rate of pancreatic cystic lesions (PCL) [1]. A substantial fraction (5-10\%) of these cystic lesions are pancreatic cystic neoplasms (PCN), $[2,3]$ a significant majority of which are mucinous neoplastic cysts including intraductal papillary mucinous neoplasm (IPMN), and mucinous cystic neoplasm (MCN). IPMN and MCN carry malignant potential [4-9]. IPMN and $\mathrm{MCN}$ are mucinous precursor lesions for pancreatic ductal adenocarcinoma (PDAC), a highly aggressive tumor currently with a very low survival rate $[10,11]$. Early detection of PDAC and its precursors has the potential to significantly increase survival rates $[12,13]$.

Accurate and early diagnosis of PCN is important for the appropriate management of patients and reduction of cancer-specific mortality. Separation of PCN from nonneoplastic PCL which are unlikely to require surveillance or surgery remains challenging. The current diagnostic approaches rely primarily on cytologic examination, tumor biomarker analysis of cyst fluid and high-resolution imaging [14]. The diagnostic power of imaging modalities including CT scan and HR-US is limited with an accuracy of 50-73\% in differentiating neoplastic from non-neoplastic cysts [15] with considerable high inter- and intra-observer variability $[16,17]$. The sensitivity of cytologic examination using EUSFNA for the detection of mucinous precursor lesions is only 49 to $59 \%$ [18]. Carcinoembryonic antigen (CEA), among other biomarkers, has the best sensitivity (59-67\%) for the detection of mucinous precursor lesions [18].

Guidelines have been published to assist in the diagnosis and management of PCL [19-21]. These incorporate clinical, laboratory and pathologic characteristics of PCL including patient age, clinical symptoms, pancreatic duct type and size, presence of a mural nodule, cytology of the cyst fluid, cyst size and diagnostic imaging characteristics [14, 19, 20,22].

Molecular changes commonly found in PDAC, including abnormalities in KRAS, GNAS, SMAD4, CDKN2A, BRAF, PIK3CA and TP53 genes can also be present in precursor lesions [23-25]. We hypothesized that incorporating the molecular analysis into PCL cytology specimens may increase the diagnostic accuracy and may help to identify patients who require surveillance or consideration for surgical management. This approach is especially important in paucicellular specimens which frequently yield a non-diagnostic cytology result yet may contain adequate material for molecular analysis $[9,26,27]$.

In the current study, we show that molecular analysis of the cyst fluid has clinical utility in identifying gene mutations associated with neoplastic PCLs and improves the sensitivity/specificity of the clinical diagnosis particularly in non-diagnostic cytology specimens.

\section{RESULTS}

\section{Molecular and cytology results for patients with surgical resections concur with algorithm-based classification}

Of 278 patients with PCL and paired molecular studies, $46(16.5 \%)$ underwent surgical resection at our institution (Supplementary Table 1). The surgical pathology diagnoses were as follows: IPMN $(n=19,41.3 \%)$ (main duct [md] - and branch duct [bd]-IPMN), MCN $(n=9$, $19.6 \%)$, PDAC $(n=8,17.4 \%)$, SCA $(n=2,4.3 \%)$, PNET $(n=3,6.5 \%)$ and pseudocysts $(n=5,10.9 \%)$ (Table 1). Among IPMN, MCN or PDAC diagnoses, cytology yielded a correct result in 15 of $36(41.6 \%)$ whereas 21 cases were classified as either non-diagnostic ("ND", $n=10$ ) or negative ("NEG", $n=11$ ). Molecular analysis of preoperative cyst fluid from the IPMN/MCN/PDAC group with 36 surgical specimens in total, showed at least one mutation in KRAS or GNAS genes in 31 (86.1\%), including 16 of the $21(76.2 \%)$ cases incorrectly classified by cytology ("NEG": $n=9$, "ND": $n=7$ ). The sensitivity of molecular analysis in detecting mucinous PCN based on the presence of any mutation was $88.9 \%$ ( 32 of 36 cases) which is significantly higher than that of cytology (41.6\%; 15 of 36 cases; $p=0.0001$, Fisher's exact test). This finding is similar to the algorithmic approach for classifying the mucinous PCL (Table 1 and Supplementary Data). Both SCA cases showed a VHL mutation. Mutations detected in cyst fluid were also detected in the surgical specimens (data not shown).

\section{Cytology diagnosis and case distribution}

Among 318 PCL cases, cytology specimens were available for 309 cases. Cytology diagnoses were classified into four groups: non-diagnostic ("ND", 28.8\%, $n=89$ ), negative for malignancy ("NEG", $50.5 \%, n=$ 156), atypical/suspicious ("ATY/SUS", $17.2 \%, n=53$,), and positive for malignancy ("POS", $3.6 \%, n=11$ ). Patient demographics are given in Table 2.

\section{Cytology alone has a limited sensitivity for mucinous PCN}

In our cohort, the algorithm identified a total of 147 mucinous PCN and 162 non-mucinous PCL (Table 2). The cytology results were compared with the algorithmbased classification. The cytology analysis alone for detecting a mucinous PCN has a sensitivity of $43.5 \%$. Of the 147 mucinous PCN with available cytology diagnoses, cytology correctly identified 64 cases with a "POS" $(n=$ $11)$ or "ATY/SUS" $(n=53)$ diagnosis but did not detect 83 cases ("ND": $n=45$, "NEG": $n=38$ ). Of 162 cases negative for mucinous PCN, cytology identified 118 cases 
Table 1: Summarizes the pathologic, molecular and cytological characteristics of 46 surgical resection specimens of pancreatic cystic lesions (PCLs).

\begin{tabular}{|c|c|c|c|c|c|c|}
\hline Cyst Type- grade & $K R A S$ & $G N A S$ & Other genes & $\begin{array}{l}\text { Cytology } \\
\text { result }\end{array}$ & $\begin{array}{c}\text { Algorithm } \\
\text { diagnosis }\end{array}$ & $\begin{array}{l}\text { Molecular } \\
\text { diagnosis" }\end{array}$ \\
\hline 1- IPMN - LG & p.G12V & NEG & NEG & NEG & mPCN & $\mathrm{mPCN}$ \\
\hline 2- IPMN - LG & p.G12V & p.R201C & NEG & $\mathbf{A} / \mathbf{S}$ & mPCN & mPCN \\
\hline 3- IPMN - LG & p.G12V & p.R201C & NEG & $\mathbf{A} / \mathbf{S}$ & mPCN & mPCN \\
\hline 4- IPMN - LG & p.G12V & p.R201H & NEG & ND & mPCN & mPCN \\
\hline 5- IPMN - LG & p.G12V & NEG & NEG & ND & mPCN & mPCN \\
\hline 6- IPMN - LG & p.G12R & NEG & NEG & $\mathbf{A} / \mathbf{S}$ & mPCN & mPCN \\
\hline 7- IPMN - LG & p.G12R & p.R201H & NEG & $\mathbf{A} / \mathbf{S}$ & mPCN & mPCN \\
\hline 8- IPMN - LG & p.G12A & p.R201H & NEG & NEG & mPCN & mPCN \\
\hline 9- IPMN - LG & p.G12R & NEG & NEG & NEG & mPCN & mPCN \\
\hline 10- IPMN - LG & NEG & NEG & NEG & ND & mPCN & nmPCL \\
\hline 11- IPMN - LG & p.G12V & p.R201C & NEG & ND & mPCN & mPCN \\
\hline 12- IPMN - LG & p.Q61H & p.R201H & NEG & ND & mPCN & mPCN \\
\hline 13- IPMN - MG & p.G12D & NEG & NEG & NEG & mPCN & mPCN \\
\hline 14- IPMN - MG & p.G12D & p.R201C & APC & NEG & mPCN & mPCN \\
\hline 15- IPMN - MG & p.G12V & p.R201C & $C D K N 2 A$ & POS & mPCN & mPCN \\
\hline 16- IPMN - MG & $\begin{array}{l}\text { p.G12A } \\
\text { p.G12T }\end{array}$ & $\begin{array}{l}\text { p.R201C } \\
\text { p.R201H }\end{array}$ & NEG & ND & mPCN & mPCN \\
\hline 17- IPMN - MG & NEG & p.R201C & NEG & ND & mPCN & mPCN \\
\hline 18- IPMN - HG & p.G12V & NEG & TP53, РIC3CA & $\mathbf{A} / \mathbf{S}$ & mPCN & mPCN \\
\hline 19- IPMN - HG & NEG & NEG & $B R A F$ & NEG & mPCN & mPCN \\
\hline 20- MCN - LG & p.Q61H & NEG & NEG & NEG & mPCN & mPCN \\
\hline 21- MCN - LG & NEG & NEG & NEG & ND & mPCN & nmPCL \\
\hline 22- MCN - LG & p.G12D & NEG & NEG & $\mathbf{A} / \mathbf{S}$ & mPCN & mPCN \\
\hline 23- MCN - LG & p.G12D & NEG & NEG & NEG & mPCN & mPCN \\
\hline 24- MCN - LG & NEG & NEG & NEG & ND & mPCN & nmPCL \\
\hline 25- MCN - MG & p.G12D & NEG & TP53 & $\mathbf{A} / \mathbf{S}$ & mPCN & mPCN \\
\hline 26- MCN - MG & p.G12V & NEG & NEG & NEG & mPCN & mPCN \\
\hline 27- MCN - MG & NEG & NEG & NEG & NEG & mPCN & nmPCL \\
\hline 28- MCN - MG & p.G12D & NEG & NEG & ND & mPCN & mPCN \\
\hline 29- PDA & p.G12R & NEG & TP53 & POS & mPCN & mPCN \\
\hline 30- PDA & p.G12V & NEG & TP53, SMAD4 & POS & mPCN & mPCN \\
\hline 31- PDA & p.G12D & NEG & SMAD4 & POS & mPCN & mPCN \\
\hline 32- PDA & p.G12D & NEG & RB1, SMAD4, PTEN & POS & mPCN & mPCN \\
\hline 33-PDA & p.G12D & NEG & TP53 & POS & mPCN & mPCN \\
\hline 34- PDA & NEG & p.R201H & SMAD4, TP53 & POS & mPCN & mPCN \\
\hline 35- PDA & p.Q61C & p.R201C & TP53, CDKN2A & POS & mPCN & mPCN \\
\hline 36- PDA & p.G12D & p.R201H & NEG & NEG & mPCN & mPCN \\
\hline 37-SCA & NEG & NEG & $V H L$ & NEG & mPCN & nmPCL \\
\hline 38-SCA & NEG & NEG & $V H L$ & NEG & nmPCL & nmPCL \\
\hline 39- PNET & NEG & NEG & NEG & $\mathbf{A} / \mathbf{S}$ & mPCN & nmPCL \\
\hline 40- PNET & NEG & NEG & ATM & POS & mPCN & nmPCL \\
\hline 41- PNET & NEG & NEG & NEG & $\mathbf{A} / \mathbf{S}$ & mPCN & nmPCL \\
\hline
\end{tabular}




\begin{tabular}{lcccccc} 
42- Pseudocyst/CP & NEG & NEG & NEG & NEG & nmPCL & nmPCL \\
43- Pseudocyst/CP & NEG & NEG & NEG & NEG & nmPCL & nmPCL \\
44- Pseudocyst/CP & p.G12D & NEG & NEG & NEG & mPCN & mPCN \\
45- Pseudocyst/CP & NEG & NEG & NEG & NEG & nmPCL & nmPCL \\
46- Pseudocyst/CP & NEG & NEG & NEG & NEG & nmPCL & nmPCL \\
\hline
\end{tabular}

(CP: chronic pancreatitis, IPMN: Intraductal papillary mucinous neoplasm, MCN: Mucinous cystic neoplasm, mPCN: mucinous pancreatic cystic neoplasm, nmPCN: non-mucinous pancreatic cystic neoplasm, PDA: Pancreatic ductal carcinoma, PNET: Pancreatic neuroendocrine tumor, SCA: Serous cystadenoma, A/S: atypical/suspicious, HG: high-grade, LG: low-grade, MG: intermediate grade, ND: non-diagnostic, NEG: Negative, POS: positive)

*Based on the presence or absence of any of the following genes: BRAF, KRAS, GNAS, PIK3CA, CDKN2A, PTEN, SMAD4, and TP53.

Table 2: Shows clinical variables of 318 cases of pancreatic cystic lesions

\begin{tabular}{|c|c|c|}
\hline Clinical variable & $\begin{array}{l}\text { Pancreatic cystic lesions } \\
\qquad(n=318)\end{array}$ & $\begin{array}{c}\text { Case Distribution: } \\
M=\text { mucinous PCN } \\
\text { NM = non-mucinous PCL }\end{array}$ \\
\hline \multicolumn{3}{|l|}{$\begin{array}{l}\text { Cytology diagnoses: } \\
\text { (n of } 309, \% \text { ) }\end{array}$} \\
\hline Non-diagnostic “ND” & $(89 / 309,28.8 \%)$ & $\mathbf{M}=45(51 \%), \mathbf{N M}=44(49 \%)(p=1.000)$ \\
\hline Negative "NEG" & $(156 / 309,50.5 \%)$ & $\mathbf{M}=38(24 \%), \mathbf{N M}=118(76 \%)(p=\mathbf{0 . 0 0 0 1})$ \\
\hline Atypical/suspicious "ATY/SUS" & $(53 / 309,17.2 \%)$ & $\mathbf{M}=53(100 \%), \mathbf{N M}=0(0 \%)(p=\mathbf{0 . 0 0 0 1})$ \\
\hline Positive " POS" & $(11 / 309,3.6 \%)$ & $\mathbf{M}=11(100 \%), \mathbf{N M}=0(0 \%)(p=\mathbf{0 . 0 0 0 1})$ \\
\hline Female : male & $162: 156(1.04)$ & $\mathbf{M}=0.87, \mathbf{N M}=1.19 \quad(p=0.18)$ \\
\hline $\begin{array}{l}\text { Patient Age (year) } \\
\text { Median (range) }\end{array}$ & $61.5,(15-93)$ & $\mathbf{M}=66.8, \mathbf{N M}=56.7 \quad(p=\mathbf{0 . 0 0 0 1})$ \\
\hline $\begin{array}{l}\text { CEA fluid concentration (ng/ml) } \\
\text { Median (range) }\end{array}$ & $1056.5,(0.1-11000)$ & $\mathbf{M}=2126.7, \mathbf{N M}=188.9(p=\mathbf{0 . 0 0 0 1})$ \\
\hline $\begin{array}{l}\text { Cyst size }(\mathrm{mm}) \\
\text { Median (range) }\end{array}$ & $33.6,(5-114)$ & $\mathbf{M}=30.2, \quad \mathbf{N M}=36.6 \quad(p=\mathbf{0 . 0 1 5})$ \\
\hline $\begin{array}{l}\text { Amylase fluid concentration }(\mathrm{u} / \mathrm{L}) \text {, } \\
\text { Median (range) }\end{array}$ & $6650.3,(2.4-24990)$ & $\mathbf{M}=5061.8, \mathbf{N M}=7764.6(p=\mathbf{0 . 0 0 8})$ \\
\hline $\begin{array}{l}\text { Cyst fluid viscosity } \\
(n=275)\end{array}$ & $\begin{array}{c}\text { Viscous }(\boldsymbol{V})=127 \\
\text { Non-viscous }(\boldsymbol{N} \boldsymbol{V})=148\end{array}$ & $\begin{array}{c}\boldsymbol{V}, \mathbf{M}=91, \mathbf{N M}=36 \\
(p=\mathbf{0 . 0 0 0 1}) \\
\mathbf{N}, \mathbf{M}=35, \mathbf{N M}=113\end{array}$ \\
\hline
\end{tabular}

The univariate analysis is performed between different characteristics in mucinous (M) and non-mucinous (NM) cases primarily classified by the proposed algorithm. Statistically significant differences are shown in bold.

as "NEG" but could not make a definitive diagnosis in 44 cases (all "ND"). While it was more likely for a cyst to be of non-mucinous subtype in the "NEG" group, the distribution of the mucinous PCN and non-mucinous PCL cases among non-diagnostic specimens were not significantly different $(p=1.00)$ (Table 2$)$.

\section{Molecular analysis}

\section{Non-diagnostic cytology specimens contain KRAS/ GNAS gene mutations}

Of cases in the "ND" group 43.7\% (38/87) showed mutations in KRAS and/or GNAS genes. This fraction is significantly higher than $K R A S$ and/or GNAS mutation frequency observed in the "NEG" group $(23.4 \%, 36 / 154, p=$ 0.002 , Table 3). By contrast, the proportion of specimens with $K R A S$ and/or GNAS mutations in "ATY/SUS" and "POS" cytology is significantly higher than the "NEG" or "ND" groups $(85.5 \%, 53 / 62, p=0.0001)$. At lower frequency, other gene mutations were also observed in "NEG" and “ND” groups [e.g TP53 (1), PIK3CA (2), ATM (2), APC (2)]. Molecular analysis failed in a total of 4 cases in all groups.

\section{Mutant allele frequency (MAF) in non- diagnostic cytology group is comparable to atypical/suspicious cytology group}

We compared the KRAS MAF (the most commonly mutated and tested gene in our cohort) between different 
Table 3: Shows KRAS/GNAS mutation prevalence across different cytology groups

\begin{tabular}{|c|c|c|c|}
\hline Cytology diagnoses & $K R A S, n(\%)$ & $G N A S, n(\%)$ & $K R A S$ or $G N A S, n(\%)$ \\
\hline Non-diagnostic "ND" & $32 / 87(36.8 \%)$ & $15 / 51(29.4 \%)$ & $38 / 87(43.7 \%)$ \\
\hline Negative "NEG" & $32 / 154(20.8 \%)$ & $14 / 81(17.3 \%)$ & $36 / 154(23.4 \%)^{*}$ \\
\hline Atypical/suspicious "ATY/SUS" & $43 / 51(84.3 \%)$ & $15 / 34(44.1 \%)$ & $44 / 51(86.3 \%)$ \\
\hline Positive "POS" & $8 / 11(72.7 \%)$ & $3 / 9(33.3 \%)$ & 9/11 (81.8\%) \\
\hline
\end{tabular}

Note: Patients with more than one mutation are represented in multiple categories.

("the presence of a gene mutation is significantly lower in the negative "NEG" group compared to other groups, see text for further details).

cytology groups. The median KRAS MAF in "ND", "NEG" and "ATY/SUS/POS" groups were 25.3\% (range; $2 \%$ $54 \%$ ), 17.8\% (range; $1.5-52.5 \%$ ) and $25.7 \%$ (range; $2 \%$ $88 \%$ ) respectively. The KRAS MAF observed in the "ND" group approached the levels detected in the "ATY/SUS/ POS" group. The difference between "NEG" and "ND" groups did not reach the statistical significance $(p=0.086)$.

ROC curve was then utilized to calculate the KRAS MAF threshold for detection of mucinous PCN defined by the algorithm (Figure 1). Cytology specimens with mutations were divided into low and high MAF groups for $K R A S$ based on the ROC-calculated threshold of $1.8 \%$. This approach has the potential to improve specificity by excluding the incidental finding of precursor lesions such as PanIN. In specimens with a KRAS mutation, a low MAF was more commonly observed in the "NEG" compared to "ND" cytology ( $p=0.02$, Fisher's exact test). There was no difference in KRAS MAF between "ND" and "ATY/ SUS/POS" $(p=1.000)$.

\section{Molecular analysis is more sensitive than cytology for detection of mucinous PCN}

The molecular analysis results of all PCL cases were compared with algorithm-based classification. Of 318 PCL cases, molecular analysis for the KRAS gene was performed in 303 cases while multi-gene analysis was performed in a subset of 182 PCL cases. Any value of KRAS MAF had a significantly higher sensitivity for mucinous PCN than cytology analysis $(82.4 \%$ vs. $43.5 \%, p=0.0001)$ without adversely affecting the specificity (93.4\%). Adding GNAS to the gene mutation analysis increased sensitivity to $93.3 \%$ (Table 4). Interestingly, 27 mucinous PCNs showed mutations in additional genes including 25 cases with additional KRAS and/or GNAS mutations (Table 5). Nevertheless, in this study expanding the molecular analysis with genes from the Ampliseq cancer hotspot panel v2 beyond $K R A S$ and GNAS did not significantly improve the detection of mucinous PCN (data not shown).

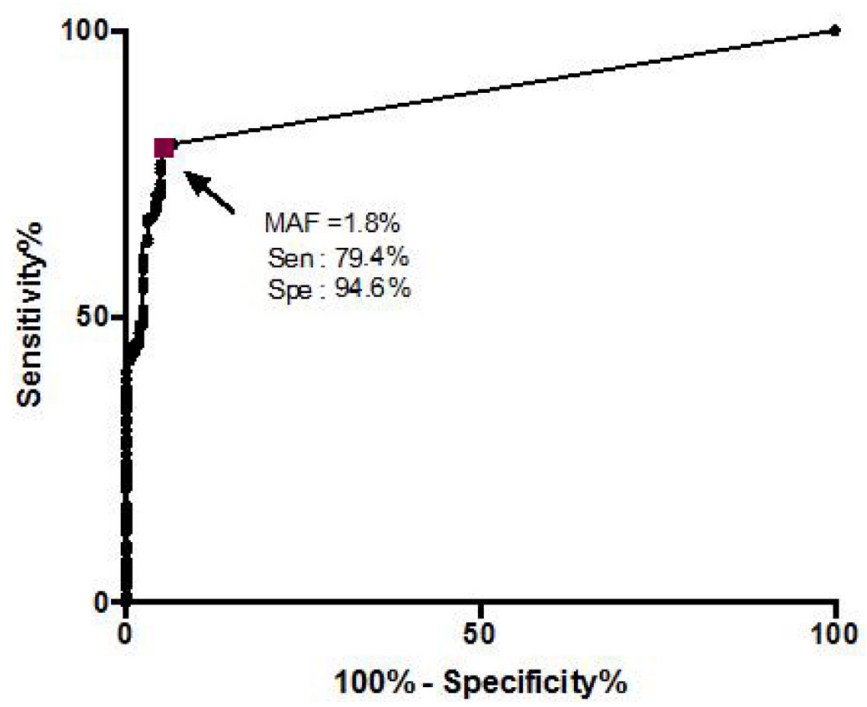

Figure 1: Receiver operating characteristic (ROC) curve plotted with $K R A S$ mutation allele frequency (MAF) in pancreatic cystic lesions classified as either mucinous or non-mucinous based on the clinical algorithm. The purple square represents the MAF of $1.8 \%$ which showed the best combination of sensitivity and specificity for discriminating mucinous and non-mucinous cysts. 
We next examined the impact of using the $1.8 \%$ cutoff to improve accuracy by separating "high" and "low" KRAS MAF. Of the non-mucinous PCL cases with a $K R A S$ mutation, 9 of $11(82 \%)$ had an MAF of more than $1.8 \%($ median $=5.3 \%$; range $=1.47-18)$. Of the mucinous PCN cases with a KRAS mutation, 106 of $117(93.2 \%)$ showed MAF of at least $1.8 \%$ (median $=26.8 \%$; range $=1.2-87.7)$. Considering only the cases with a "high" $K R A S$ MAF as mucinous PCN, a marginal change in the sensitivity $(82.4 \%$ to $79.5 \%)$ and specificity $(92.4 \%$ to $94.6 \%$ ) was observed.

\section{Multiple mutations are common in pancreatic cystic lesions}

Of PCL tested for GNAS and KRAS gene mutations, $19.8 \%(36 / 182)$ showed both KRAS and GNAS mutations. Thirty-five (97\%) cases were predicted to have a mucinous PCN based on the algorithm-based classification. Among cases with double mutations, 33.3\% (12/36) have at least $5 \%$ difference in MAF of two different genes. Of these, $83 \%(10 / 12)$ had a higher KRAS MAF $(p=0.003)$. In addition, $11 \%(14 / 127)$ of mucinous PCN cases carried more than one mutation in the same gene (KRAS: $10.4 \%$ [12/115], GNAS: $6.3 \%$ [3/47]). In 7 of 15 cases, the MAF differed by at least $5 \%$. These findings may be due to presence of multiple cysts, or tumor heterogeneity or clonal evolution within a cyst (e.g. KRAS followed by GNAS mutation).

\section{The number and frequency of oncogenic mutations correlate with dysplasia}

Of all the cases with available surgical resections, 36 cases were either pancreatic adenocarcinoma (PDAC, $n=8$ ) or one of its precursors (IPMN, $\mathrm{n}=19$ and MCN, $n=9$ ) (Table 1). Of 36 cases $17(47 \%)$ were low grade (LG) lesions (IPMN, $n=12$ and $\mathrm{MCN}, n=5$ ). These cases showed only $\operatorname{KRAS}(n=14)$ and/or GNAS $(n=$ 7) mutations. No double KRAS or double GNAS gene mutation was observed in these LG lesions. Of 9 cases $(25 \%)$ with intermediate grade dysplasia (IPMN, $n=5$ and $\mathrm{MCN}, n=4)$, three (30\%) showed mutations in three additional genes (TP53, CDKN2A, APC). This group also had one case with double mutation in both $K R A S$ and GNAS (case \#16, Table 1). All PDAC and mucinous PCN cases with high grade dysplasia $(n=10)$, showed additional gene mutations (TP53, SMAD4, CDKN2A, BRAF, ARID1A, RB1, PTEN, PIK3CA).

\section{DISCUSSION}

Incorporation of multiple diagnostic modalities such as imaging techniques, cyst fluid chemical analysis and clinical findings, although helpful, do not provide early detection or accurate differentiation between non- neoplastic and neoplastic pancreatic lesions [15, 28]. Although rare, some mucinous PCNs carry a relatively high risk of malignancy. There remains a critical need for a better diagnostic tool to stratify patients for proper clinical management [29]. Recent studies indicate that molecular analysis of the KRAS and GNAS genes may be helpful in classifying PCL into neoplastic and nonneoplastic categories [30, 31]. Although many of these recent studies showed the added benefit of presence of molecular alterations in oncogenic driver genes, such as $K R A S$, the molecular analysis has not yet found its way into the standardized guidelines of pancreatic cystic lesions [32]. Here, we demonstrate the value of molecular analysis in patients with neoplastic PCL yet classified as "negative" or "non-diagnostic" by cytology. In a large cohort of PCL, we confirmed that cytologic evaluation has limited diagnostic value for low grade PCN [18]. In addition, we show that expanding the molecular panel beyond the KRAS and GNAS genes was helpful in identifying mucinous PCNs with moderate to high grade dysplasia.

Considering the surgical cases with mucinous PCN, there were a substantial number of cases $(27.8 \% ; 10$ of 36 ) with non-diagnostic cytology. This limitation is similar to the previously published studies contributing to the low diagnostic sensitivity of cytology [33]. In contrast, molecular analysis showed a sensitivity of $88.9 \%$ (32 of 36). Furthermore, $90 \%$ of surgically proven PCN with non-diagnostic cytology showed at least one mutation in $K R A S$ and/or GNAS genes (Table 1). The presence of these mutations and the MAF were significantly higher in the paucicellular non-diagnostic category than in the negative cytology group. These results suggest the molecular analysis is dependent on cell-free DNA, rather than cyst-fluid cellularity, and can serve as an important complement to morphologic evaluation particularly for pancreatic cystic lesions with non-diagnostic cytology.

In this study, we used an algorithmic approach based on international consensus guidelines to classify mucinous PCN and non-mucinous PCL [14, 19, 21, 34]. Surgical specimens $(46 / 278,16.5 \%)$ were used to assess the accuracy of the algorithm in classifying the PCL. Final pathologic diagnoses of surgical specimens were in agreement with the algorithm ( $89 \%$ concordance). When surgical specimens were used as the gold standard for the diagnosis of mucinous PCN, the sensitivity of cytology and molecular analysis was $41.7 \%$ and $88.9 \%$, respectively. Cytology and molecular analysis of the cohort of 318 specimens classified using the algorithmic approach produced similar findings, yielding a sensitivity of $43.5 \%$ and $93.3 \%$, respectively (table 4 ). To better assess the diagnostic value of cytology, paired analysis with surgical resection would be optimal; however, few patients need radical surgery. Nevertheless, our data support the use of the algorithm to classify PCL into mucinous and non-mucinous categories [35]. 
Table 4: Illustrates the comparison between molecular analysis (KRAS or KRAS/GNAS) and cytology analysis for detection of a precursor mucinous pancreatic cystic lesion

\begin{tabular}{lccc}
\hline Gene & $\boldsymbol{K R} \boldsymbol{A} \boldsymbol{S}$ & $\boldsymbol{K R} \boldsymbol{A S}$ or $\boldsymbol{G N A S}$ & Cytology analysis \\
\hline Number of cases & $\mathbf{( 3 0 3 )}$ & $(\mathbf{1 8 2})^{\mathrm{a}}$ & $(\mathbf{3 0 9 )}$ \\
Sensitivity: & $82.4 \%^{*}$ & $93.3 \%^{*}$ & $43.5 \%$ \\
Specificity: & $93.4 \%$ & $83.9 \%$ & $100 \%$ \\
PPV: & $90.7 \%$ & $84.7 \%$ & $100 \%$ \\
NPV: & $87.0 \%$ & $92.9 \%$ & $65.9 \%$ \\
\hline
\end{tabular}

"Sensitivity of molecular analysis is significantly higher than cytology $(p=0.0001)$.

${ }^{a} 182$ cases were tested by NGS for both KRAS and GNAS genes.

Table 5: Summarizes the non $K R A S / G N A S$ mutations found in precursor lesions

\begin{tabular}{lc}
\hline Mutation type & Number of cases with mutation \\
\hline$V H L$ & 8 \\
$P I K 3 C A, T P 53$ & 6 \\
$A T M$ & 5 \\
$S M A D 4$ & 4 \\
$C D K N 2 A$ & 3 \\
$A P C, F G F R 3, J A K 3$ & 2 \\
$R B 1, A K T 1$, ARID1A, MET, FBXW7, ERBB4, PTEN, FGFR2, KIT, SMO & \multicolumn{2}{|c}{1} \\
\hline
\end{tabular}

The cases with $V H L$ gene mutation are likely to be serous cyst adenomas (SCA).

The algorithm disagreed with molecular analysis results in several cases. Eleven cases classified as nonmucinous PCL by the algorithm, showed KRAS $(n=$ $10)$ or $\operatorname{GNAS}(n=1)$ mutations. To improve accuracy of molecular analysis, we applied the cut-off of $1.8 \%$ KRAS MAF obtained from the ROC analysis. However, this cutoff value reclassified only 2 of 11 cases as nonmucinous PCL. While it is possible that these specimens represent true mucinous $\mathrm{PCN}$ misclassified by the algorithm, no follow-up surgical resection was available to support this possibility. In addition, 11 cases classified as mucinous PCN had KRAS MAF of less than 1.8\%. These results suggest that use of a MAF cutoff for KRAS mutation may have a modest impact on specificity.

Our study demonstrates that gene mutation analysis can help discriminate between non-neoplastic and neoplastic cystic lesions. KRAS and GNAS gene analysis provided an overall sensitivity for detection of mucinous PCN, which was significantly higher than cytology alone (93.3\% vs. $43.5 \%$, Table 4$)$. In the absence of KRAS or GNAS mutations, inclusion of genes such as TP53, PTEN, SMAD4, PIK3CA, CDKN2A, BRAF and RBI in the molecular analysis identified only one additional mucinous PCN. Although this finding suggests expanded gene panels may have limited utility for initial diagnosis, analysis of additional genes such as RNF43, ATRX and $D A X X$, which were not included in our study, may further improve diagnostic sensitivity $[35,36]$.
Regardless, an expanded gene panel may also be informative for dysplasia. Notably, among patients with surgical specimens, mucinous PCN with low-grade dysplasia showed mutations only in $K R A S$ and/or GNAS genes. In contrast, mutations in additional genes were observed in mucinous PCN with moderate $(3 / 9,33 \%)$ and high grade $(9 / 10,90 \%)$ dysplasia. Both the number and the MAF of the of mutated genes increased with the grade of dysplasia (Table 1). This finding suggests acquisition of additional mutations is associated with progression from low grade to high grade dysplasia [37, 38]. The same mutations were detected in paired cytology and surgical specimens. Consequently, detection of such mutations in cytology samples, particularly in non-diagnostic specimens, may indicate the presence of a higher grade lesion and may warrant consideration for resampling or for surgical intervention in the appropriate context [35, 39]. For instance, MCN and IPMN with high grade dysplasia both require surgical excision; whereas low grade lesions may need more conservative approach, such as periodic observation for possible progression [40]. As none of the low grade mucinous PCN in our study had more than one KRAS and/ or GNAS mutations, in the absence of other indications (e.g. grave clinical symptoms, positive cytology), periodic monitoring may be sufficient (Table 1). The absence of any mutations, nevertheless, did not preclude the need for surgery, since this molecular panel is not comprehensive and failed to detect PNET and 3 of $9 \mathrm{MCN}$. 
Our study has both strengths and limitations. The incorporation of cytologic diagnosis into the algorithm [14] may artificially increase the specificity of cytology in this study. By contrast, molecular analysis was not included in the algorithm and consequently the sensitivity and specificity of molecular results are not affected. The choice of the cyst CEA level may also influence the accuracy of the algorithm. Based on previous studies, we used a CEA level of $192 \mathrm{ng} / \mathrm{ml}$ as a threshold for the classification of mucinous PCL [41, 42]. In our study ROC analysis for cyst CEA levels suggested that the cut off value of $124 \mathrm{ng} / \mathrm{ml}$, rather than $192 \mathrm{ng} /$ $\mathrm{ml}$, would have the best combination of sensitivity and specificity for discriminating between mucinous and non-mucinous PCL (supplemental data). Nevertheless, the high concordance observed between the algorithm and surgical pathology diagnosis supports the use 192 $\mathrm{ng} / \mathrm{ml}$ threshold.

This cohort was followed for a median of 3.3 years (range 1-8 years). Interestingly, 149 of the 318 specimens were classified as mucinous PCN by the algorithm, yet only 36 patients with mucinous PCN had surgical resection. In our study, it is possible that some patients may have been lost to follow up, since 12 patients had other mutations (i.e. $A K T 1, A P C, C D K N 2 A$, FGFR2/3, PIK3CA SMAD4 or TP53) in addition to $K R A S$ and/or GNAS mutations. Six of these patients had atypical/suspicious cytology diagnosis. Nevertheless, these results are consistent with the current guidelines $[14,21]$ and support the proposal that many mucinous $\mathrm{PCN}$ do not require surgical intervention at the time of diagnosis [35].

In summary, the high accuracy of somatic molecular analysis of pancreatic cyst fluid, particularly with nondiagnostic cytology specimens, to identify patients with neoplastic PCN which are more likely to benefit from surveillance, suggests that the standard of care should include somatic mutation analysis in the evaluation of pancreatic cystic lesions.

\section{MATERIALS AND METHODS}

\section{Case selection}

This study (IRB 14071) was approved by the institutional review boards of the University of Massachusetts Memorial Medical Center, Worcester, MA. From January 2008 to October 2015, 318 EUS-guided FNA of cyst fluid from 278 patients with pancreatic cyst(s) was evaluated during routine clinical testing, with follow up until December 2016. Nine specimens were not submitted for cytology analysis. Six specimens were not submitted for or failed molecular studies. Cyst fluid was subjected to chemical, cytopathologic and molecular genetic studies. Clinical, radiological and surgical followup data, if available, were recorded.

\section{Chemical analysis}

The cyst fluid CEA $(n=224)$ levels were tested in most patients based on the strong clinical suspicion for PCN (Immunoassay (IA) - Quest Diagnostics ${ }^{\mathrm{TM}}$ ). The concurrent serum levels were also available for a select group of patients.

\section{Cytopathologic analysis}

Cytology slides, prepared from the cyst fluid, were stained with the Papanicolaou (Pap) stain [43]. A DiffQuik stain was also performed on air-dried smears. Based upon the cytology diagnosis, the cases were categorized into four groups: non-diagnostic (paucicellular or acellular), negative for malignancy, atypical/suspicious for malignancy and positive for malignancy [44]. To provide sufficient statistical power the atypical/suspicious/ positive cases were grouped together for mutation allele frequency (MAF) analysis. These diagnostic categories are commonly used for pancreatic solid lesions. Although this multitiered reporting system can be applied to cystic lesions, it may be less reproducible due to heterogeneity and pauci-cellularity [44]. The diagnostic imaging, cyst fluid biomarker and endoscopic results and clinical impression were accessible prior to the cytopathologists' diagnosis whereas the molecular studies were not.

\section{Molecular analysis}

\section{DNA extraction}

To extract DNA from pancreatic cystic fluid, QIAamp Circulating Nucleic Acid Kit 55114 (QIAGEN Inc., Valencia, CA) was used. Extracted DNA was quantified with a UV NanoDrop 1000 spectrophotometer (DNA/RNA Calculator, Thermo Scientific, Waltham, MA) to determine the A260/A280 ratio. DNA quality was also evaluated using endpoint multiplex PCR to ensure DNA fragmentation was minimal. To extract DNA from surgical specimens, areas of tumor were identified by pathologists (AS, EFC, PAM) and manually micro-dissected from formalin-fixed, paraffinembedded tissue. The collected cells were digested and genomic DNA was extracted as previously described [45].

\section{Peptide Nucleic Acid (PNA) clamp PCR}

Specific primer sets were used to amplify the region of the KRAS gene containing codons 12, 13 and 61, GNAS gene containing codon 201 and $B R A F$ gene containing codons 598-602. PCR reactions were performed with and without a PNA clamp designed to block amplification of wild-type sequences (Supplementary Figure 1). In this real time PCR assay, the number of PCR cycles between the PCR reaction with and without PNA clamp was calculated to obtain a delta $\mathrm{Ct}(\Delta \mathrm{Ct})$. If the $\Delta \mathrm{Ct}$ is $\geq 2$ cycles above the $1 \%$ control, the result is reported as wild-type. If $\Delta \mathrm{Ct}$ is $<2$ 
cycles from the $1 \%$ control, the result is reported as mutant after BigDye Sanger sequencing confirmation (Thermo Fisher Scientific Inc., Waltham, MA). Comparing the value of $\Delta \mathrm{Ct}$ in positive control/negative control $(100 \%$, $10 \%, 1 \%$ and $0 \%$ of mutation, respectively) with that of a patient specimen can be used to quantify the mutation levels i.e. if the $\Delta \mathrm{Ct}$ equals the value of $100 \%$ positive control, the mutation frequency is $100 \%$. PCR amplicons from the PNA reaction were digested with ExoSAP-IT ${ }^{\circledR}$ [46]. DNA products were then purified from the Sanger sequencing reaction and analyzed using capillary gel electrophoresis (ABI 3500xL Genetic Analyzer, Applied Biosystems, Foster City, CA) and fluorescence detection. This mutation analysis is able to detect the wild-type sequence and all known mutations associated with tested codons [47]. Results were interpreted using Soft Genetics Mutation Surveyor (SoftGenetics, State College, PA). The detection limit of the diluted positive control DNA in a background of wild-type alleles was $0.01 \%$.

\section{Next Generation Sequencing (NGS)}

Next generation sequencing was performed as previously described using Ampliseq cancer hotspot panel V2 [48]. Amplicon libraries were created from genomic DNA (10 ng) according to the manufacturer's protocol (Ion AmpliSeq Library Kit 2.0, Thermo Fisher Scientific Inc). The Ion CHEF Template System (Thermo Fisher Scientific Inc.) was used for emulsion PCR to amplify library DNA onto IonSphere Particles (ISPs) and load 318v2 chips. Sequencing was performed on an Ion Torrent PGM (IC200 Sequencing Kit, Thermo Fisher Scientific Inc. Waltham, MA) with coverage of 500-2500X. Variant calls from two software pipelines, Variant Caller V5 (Life Technologies, Inc) and NextGene V2 (SoftGenetics, Inc) were compared using a laboratory developed visual basic excel program. Variants with $\geq 10$ reads and allele frequency greater than $1 \%$ were called positive although the limit of sensitivity is $0.2 \%$. A manual review of nucleotide sequences was also performed for the genes frequently mutated in pancreatic cysts. After review, variants were confirmed as somatic mutations in the Catalogue of Somatic Mutations in Cancer (COSMIC) database [49] or ruled out as a known germline single-nucleotide polymorphisms (SNP) with the dbSNP database [50].

\section{Algorithm to identify mucinous neoplastic cysts}

Cytologic diagnosis, cyst fluid CEA levels, cyst size, the presence of a mural nodule, fluid viscosity, and EUS clinical impression were used in a step-wise algorithmic approach to discriminate between mucinous and nonmucinous PCLs (supplemental data and Supplementary Figure 2 for further details).

\section{Receiver operating characteristics (ROC) curve analysis}

ROC analysis was used to separate mucinous PCN from non-mucinous cysts based on 1) "high" and "low" KRAS MAF (defined as relative fraction of mutant PCR amplicon in the total DNA amplicon [51] and 2) cyst fluid CEA (see Supplemental Data) levels ( $\mathrm{ng} / \mathrm{ml}$ ).

The KRAS MAF from cysts classified by the algorithm as non-mucinous $(n=165)$ and mucinous $(n=$ 146) was plotted on an ROC curve to define a KRAS MAF threshold to most accurately separate non-mucinous from mucinous neoplastic lesion.

\section{Statistical analysis}

Analysis of statistical difference between categorical data in our study was performed based on two-tailed Fisher's exact test. Numerical data was analyzed using the Mann-Whitney Exact Test. Sensitivity, specificity, positive predictive value, and negative predictive value were calculated using standard $2 \times 2$ contingency tables. $p$ value of less than 0.05 is considered significant. ROC calculation is performed using GraphPad PRISM 7 software.

\section{Abbreviations}

CEA: carcinoembryonic antigen; CT: computed tomography; COSMIC: Catalogue of Somatic Mutations in Cancer; EUS-FNA: endoscopic ultrasound-guided fine needle aspiration; HR-US: high resolution ultrasonography; MRI: magnetic resonance imaging; MAF: mutation allele frequency; NGS: Next Generation Sequencing; PCL: pancreatic cystic lesions; PCN: pancreatic cystic neoplasms; PNET: pancreatic neuroendocrine tumors; Pap: Papanicolaou; PNA: Peptide Nucleic Acid; ROC: Receiver Operating Characteristics; SNP: Single-nucleotide polymorphisms; IPMN: intraductal papillary mucinous neoplasms; MCN: mucinous cystic neoplasms; PanIN: pancreatic intraepithelial neoplasia; SPN: solid pseudopapillary neoplasms; SCT: serous cystic tumors; PDAC: pancreatic ductal adenocarcinoma; ND: non-diagnostic; NEG: negative for malignancy; A/S: atypical/suspicious; POS: positive for malignancy.

\section{Author contributions}

Ali Sakhdari: Study design, Acquisition, Assembly, Analysis and interpretation of all clinical data, Concept of algorithm, statistical analysis, drafting of manuscript. Parnian Ahmadi Moghaddam: Acquisition, Assembly of clinical data, drafting of manuscript, critical revision of the manuscript for important intellectual content. Chi Young Ok: Acquisition, Analysis and interpretation of clinical data, drafting of manuscript, critical revision 
of the manuscript for important intellectual content. Otto Walter: Analysis and interpretation of data. Keith Tomaszewicz: KRAS and NGS assay design, Generation and analysis of KRAS/GNAS results, technical support. Mandi-Lee Caporelli: Generation and analysis of KRAS/ NGS results, technical support Xiuling Meng: Generation and analysis of KRAS/NGS results, technical support. Jennifer LaFemina: Study concept, Acquisition of surgical specimens, critical revision of the manuscript for important intellectual content. Giles Whalen: Study concept, obtained funding from pancreatic cancer alliance, Acquisition of surgical specimens, review of the manuscript. Edward Belkin: Study design and IRB, Acquisition, Assembly of clinical data, Acquisition of cyst fluid. Jaroslav Zivny Acquisition of cyst fluid, review of the manuscript. Wahid Wassef: Conception and Study design and IRB, Acquisition of cyst fluid, Study supervision. Bruce A. Woda: Obtained funding from the Department of Pathology, Clinical Interpretation of molecular studies, and critical revision of the manuscript for important intellectual content. Lloyd M. Hutchinson: Study design and IRB, Study supervision, drafting of manuscript, Concept of algorithm, Analysis of molecular results, clinical data and statistical analysis. Ediz F. Cosar: Study design, Study supervision, drafting of manuscript, Clinical Interpretation of cytology and molecular studies.

\section{ACKNOWLEDGMENTS AND FUNDING}

We acknowledge the Pancreatic Cancer Alliance for their financial support enabling the Laboratory of Diagnostic Molecular Oncology to develop Next Generation Sequencing. Pancreatic Cancer Alliance (www. pancreaticalliance.org). University of Massachusetts Department of Pathology. We acknowledge Karen Dresser for technical support and members of our UMASS laboratory for clinical support.

\section{CONFLICTS OF INTEREST}

The authors have no potential conflicts (financial, professional or personal) to report.

\section{REFERENCES}

1. Garcea G, Ong SL, Rajesh A, Neal CP, Pollard CA, Berry DP, Dennison AR. Cystic lesions of the pancreas. A diagnostic and management dilemma. Pancreatology. 2008; 8:236-51. https://doi.org/10.1159/000134279. [PubMed]

2. Centeno BA. Fine needle aspiration biopsy of the pancreas. Clin Lab Med. 1998; 18:401-27, v-vi. https://doi. org/10.1016/S0272-2712(18)30157-4. [PubMed]

3. Brugge WR, Lauwers GY, Sahani D, Fernandez-del Castillo C, Warshaw AL. Cystic neoplasms of the pancreas. N
Engl J Med. 2004; 351:1218-26. https://doi.org/10.1056/ NEJMra031623. [PubMed]

4. Kobari M, Egawa S, Shibuya K, Shimamura H, Sunamura M, Takeda K, Matsuno S, Furukawa T. Intraductal papillary mucinous tumors of the pancreas comprise 2 clinical subtypes: differences in clinical characteristics and surgical management. Arch Surg. 1999; 134:1131-36. https://doi. org/10.1001/archsurg.134.10.1131. [PubMed]

5. Salvia R, Fernández-del Castillo C, Bassi C, Thayer SP, Falconi M, Mantovani W, Pederzoli P, Warshaw AL. Main-duct intraductal papillary mucinous neoplasms of the pancreas: clinical predictors of malignancy and long-term survival following resection. Ann Surg. 2004; 239:67885. https://doi.org/10.1097/01.sla.0000124386.54496.15. [PubMed]

6. Salvia R, Festa L, Butturini G, Tonsi A, Sartori N, Biasutti C, Capelli P, Pederzoli P. Pancreatic cystic tumors. Minerva Chir. 2004; 59:185-207. [PubMed]

7. Sugiyama M, Izumisato Y, Abe N, Masaki T, Mori T, Atomi Y. Predictive factors for malignancy in intraductal papillarymucinous tumours of the pancreas. Br J Surg. 2003; 90:1244-49. https://doi.org/10.1002/bjs.4265. [PubMed]

8. Rockacy M, Khalid A. Update on pancreatic cyst fluid analysis. Ann Gastroenterol. 2013; 26:122-27. [PubMed]

9. Gardner TB, Glass LM, Smith KD, Ripple GH, Barth RJ, Klibansky DA, Colacchio TA, Tsapakos MJ, Suriawinata AA, Tsongalis GJ, Pipas JM, Gordon SR. Pancreatic cyst prevalence and the risk of mucin-producing adenocarcinoma in US adults. Am J Gastroenterol. 2013; 108:1546-50. https://doi.org/10.1038/ajg.2013.103. [PubMed]

10. Vincent A, Herman J, Schulick R, Hruban RH, Goggins M. Pancreatic cancer. Lancet. 2011; 378:607-20. https://doi. org/10.1016/S0140-6736(10)62307-0. [PubMed]

11. Siegel R, Ma J, Zou Z, Jemal A. Cancer statistics, 2014. CA Cancer J Clin. 2014; 64:9-29. https://doi.org/10.3322/ caac. 21208. [PubMed]

12. Chari ST, Kelly K, Hollingsworth MA, Thayer SP, Ahlquist DA, Andersen DK, Batra SK, Brentnall TA, Canto M, Cleeter DF, Firpo MA, Gambhir SS, Go VL, et al. Early detection of sporadic pancreatic cancer: summative review. Pancreas. 2015; 44:693-712. https://doi.org/10.1097/ MPA.0000000000000368. [PubMed]

13. Lennon AM, Wolfgang CL, Canto MI, Klein AP, Herman JM, Goggins M, Fishman EK, Kamel I, Weiss MJ, Diaz LA, Papadopoulos N, Kinzler KW, Vogelstein B, Hruban RH. The early detection of pancreatic cancer: what will it take to diagnose and treat curable pancreatic neoplasia? Cancer Res. 2014; 74:3381-89. https://doi.org/10.1158/0008-5472. CAN-14-0734. [PubMed]

14. Tanaka M, Fernández-del Castillo C, Adsay V, Chari S, Falconi M, Jang JY, Kimura W, Levy P, Pitman MB, Schmidt CM, Shimizu M, Wolfgang CL, Yamaguchi K, Yamao K, and International Association of Pancreatology. International consensus guidelines 2012 for the management 
of IPMN and MCN of the pancreas. Pancreatology. 2012; 12:183-97. https://doi.org/10.1016/j.pan.2012.04.004. [PubMed]

15. Fisher WE, Hodges SE, Yagnik V, Morón FE, Wu MF, Hilsenbeck SG, Raijman IL, Brunicardi FC. Accuracy of $\mathrm{CT}$ in predicting malignant potential of cystic pancreatic neoplasms. HPB (Oxford). 2008; 10:483-90. https://doi. org/10.1080/13651820802291225. [PubMed]

16. Ahmad NA, Kochman ML, Lewis JD, Ginsberg GG. Can EUS alone differentiate between malignant and benign cystic lesions of the pancreas? Am J Gastroenterol. 2001; 96:3295-300. $\quad$ https://doi.org/10.1111/j.15720241.2001.05328.x. [PubMed]

17. Cizginer S, Turner BG, Bilge AR, Karaca C, Pitman MB, Brugge WR. Cyst fluid carcinoembryonic antigen is an accurate diagnostic marker of pancreatic mucinous cysts. Pancreas. 2011; 40:1024-28. https://doi.org/10.1097/ MPA.0b013e31821bd62f. [PubMed]

18. Thornton GD, McPhail MJ, Nayagam S, Hewitt MJ, Vlavianos P, Monahan KJ. Endoscopic ultrasound guided fine needle aspiration for the diagnosis of pancreatic cystic neoplasms: a meta-analysis. Pancreatology. 2013; 13:48-57. https://doi.org/10.1016/j.pan.2012.11.313. [PubMed]

19. Tanaka M, Chari S, Adsay V, Fernandez-del Castillo C, Falconi M, Shimizu M, Yamaguchi K, Yamao K, Matsuno $\mathrm{S}$, and International Association of Pancreatology. International consensus guidelines for management of intraductal papillary mucinous neoplasms and mucinous cystic neoplasms of the pancreas. Pancreatology. 2006; 6:17-32. https://doi.org/10.1159/000090023. [PubMed]

20. Ohtsuka T, Matsunaga T, Kimura H, Watanabe Y, Tamura K, Ideno N, Aso T, Miyasaka Y, Ueda J, Takahata S, Osoegawa $\mathrm{T}$, Igarashi $\mathrm{H}$, Ito $\mathrm{T}$, et al. Role of pancreatic juice cytology in the preoperative management of intraductal papillary mucinous neoplasm of the pancreas in the era of international consensus guidelines 2012. World J Surg. 2014; 38:2994 3001. https://doi.org/10.1007/s00268-014-2684-y. [PubMed]

21. Elta GH, Enestvedt BK, Sauer BG, Lennon AM. ACG Clinical Guideline: Diagnosis and Management of Pancreatic Cysts. Am J Gastroenterol. 2018; 113:464-79. https://doi.org/10.1038/ajg.2018.14. [PubMed]

22. Brugge WR. Cyst fluid: moving beyond the carcinoembryonic antigen. Gastrointest Endosc. 2015; 82:1070-71. https://doi. org/10.1016/j.gie.2015.08.005. [PubMed]

23. Kanda M, Matthaei H, Wu J, Hong SM, Yu J, Borges M, Hruban RH, Maitra A, Kinzler K, Vogelstein B, Goggins M. Presence of somatic mutations in most early-stage pancreatic intraepithelial neoplasia. Gastroenterology. 2012; 142:730 733.e9. https://doi.org/10.1053/j.gastro.2011.12.042. [PubMed]

24. Maitra A, Fukushima N, Takaori K, Hruban RH. Precursors to invasive pancreatic cancer. Adv Anat Pathol. 2005; 12:81-91. https://doi.org/10.1097/01. pap.0000155055.14238.25. [PubMed]

25. Hruban RH, Takaori K, Klimstra DS, Adsay NV, AlboresSaavedra J, Biankin AV, Biankin SA, Compton C, Fukushima
N, Furukawa T, Goggins M, Kato Y, Klöppel G, et al. An illustrated consensus on the classification of pancreatic intraepithelial neoplasia and intraductal papillary mucinous neoplasms. Am J Surg Pathol. 2004; 28:977-87. https://doi. org/10.1097/01.pas.0000126675.59108.80. [PubMed]

26. Frampton AE, Stebbing J, Gall TM, Silver B, Jiao LR, Krell J. Activating mutations of GNAS and KRAS in cystic fluid can help detect intraductal papillary mucinous neoplasms of the pancreas. Expert Rev Mol Diagn. 2015; 15:325-28. https://doi.org/10.1586/14737159.2015.10027 71. [PubMed]

27. Bellizzi AM, Stelow EB. Pancreatic cytopathology: a practical approach and review. Arch Pathol Lab Med. 2009; 133:388-404. https://doi.org/10.1043/1543-2165133.3.388. [PubMed]

28. Das A, Brugge W, Mishra G, Smith DM, Sachdev M, Ellsworth E. Managing incidental pancreatic cystic neoplasms with integrated molecular pathology is a costeffective strategy. Endosc Int Open. 2015; 3:E479-86. https://doi.org/10.1055/s-0034-1392016. [PubMed]

29. Giovannetti E, Funel N, Peters GJ, Del Chiaro M, Erozenci LA, Vasile E, Leon LG, Pollina LE, Groen A, Falcone A, Danesi R, Campani D, Verheul HM, Boggi U. MicroRNA-21 in pancreatic cancer: correlation with clinical outcome and pharmacologic aspects underlying its role in the modulation of gemcitabine activity. Cancer Res. 2010; 70:4528-38. https://doi.org/10.1158/0008-5472. CAN-09-4467. [PubMed]

30. Nikiforova MN, Khalid A, Fasanella KE, McGrath KM, Brand RE, Chennat JS, Slivka A, Zeh HJ, Zureikat AH, Krasinskas AM, Ohori NP, Schoedel KE, Navina S, et al. Integration of KRAS testing in the diagnosis of pancreatic cystic lesions: a clinical experience of 618 pancreatic cysts. Mod Pathol. 2013; 26:1478-87. https://doi.org/10.1038/ modpathol.2013.91. [PubMed]

31. Rosenbaum MW, Jones M, Dudley JC, Le LP, Iafrate AJ, Pitman MB. Next-generation sequencing adds value to the preoperative diagnosis of pancreatic cysts. Cancer Cytopathol. 2017; 125:41-47. https://doi.org/10.1002/ cncy.21775. [PubMed]

32. Del Chiaro M, Besselink MG, Scholten L, Bruno MJ, Cahen DL, Gress TM, van Hooft JE, Lerch MM, Mayerle J, Hackert T, Satoi S, Zerbi A, Cunningham D, et al, and European Study Group on Cystic Tumours of the Pancreas. European evidence-based guidelines on pancreatic cystic neoplasms. Gut. 2018; 67:789-804. https://doi.org/10.1136/ gutjnl-2018-316027. [PubMed]

33. Lim LG, Lakhtakia S, Ang TL, Vu CK, Dy F, Chong VH, Khor CJ, Lim WC, Doshi BK, Varadarajulu S, Yasuda K, Wong JY, Chan YH, et al, and Asian EUS Consortium. Factors determining diagnostic yield of endoscopic ultrasound guided fine-needle aspiration for pancreatic cystic lesions: a multicentre Asian study. Dig Dis Sci. 2013; 58:1751-57. https://doi.org/10.1007/s10620-012-2528-2. [PubMed] 
34. Hsiao CY, Yang CY, Wu JM, Kuo TC, Tien YW. Utility of the 2006 Sendai and 2012 Fukuoka guidelines for the management of intraductal papillary mucinous neoplasm of the pancreas: A single-center experience with 138 surgically treated patients. Medicine (Baltimore). 2016; 95:e4922. https://doi.org/10.1097/MD.0000000000004922. [PubMed]

35. Springer S, Wang Y, Dal Molin M, Masica DL, Jiao Y, Kinde I, Blackford A, Raman SP, Wolfgang CL, Tomita T, Niknafs N, Douville C, Ptak J, et al. A combination of molecular markers and clinical features improve the classification of pancreatic cysts. Gastroenterology. 2015; 149:1501-10. https://doi.org/10.1053/j.gastro.2015.07.041. [PubMed]

36. Layfield LJ, Ehya H, Filie AC, Hruban RH, Jhala N, Joseph L, Vielh P, Pitman MB. Utilization of ancillary studies in the cytologic diagnosis of biliary and pancreatic lesions: The Papanicolaou Society of Cytopathology Guidelines. Cytojournal. 2014; 11:4. https://doi.org/10.4103/17426413.133352. [PubMed]

37. Conner JR, Mariño-Enríquez A, Mino-Kenudson M, Garcia E, Pitman MB, Sholl LM, Srivastava A, Doyle LA. Genomic Characterization of Low- and HighGrade Pancreatic Mucinous Cystic Neoplasms Reveals Recurrent KRAS Alterations in "High-Risk" Lesions. Pancreas. 2017; 46:665-71. https://doi.org/10.1097/ MPA.0000000000000805. [PubMed]

38. Jones M, Zheng Z, Wang J, Dudley J, Albanese E, Kadayifci A, Dias-Santagata D, Le L, Brugge WR, Fernandez-del Castillo C, Mino-Kenudson M, Iafrate AJ, Pitman MB. Impact of next-generation sequencing on the clinical diagnosis of pancreatic cysts. Gastrointest Endosc. 2016; 83:140-48. https://doi.org/10.1016/j.gie.2015.06.047. [PubMed]

39. Singhi AD, Zeh HJ, Brand RE, Nikiforova MN, Chennat JS, Fasanella KE, Khalid A, Papachristou GI, Slivka A, Hogg M, Lee KK, Tsung A, Zureikat AH, et al. American Gastroenterological Association guidelines are inaccurate in detecting pancreatic cysts with advanced neoplasia: a clinicopathologic study of 225 patients with supporting molecular data. Gastrointest Endosc. 2016; 83:1107-1117. e2. https://doi.org/10.1016/j.gie.2015.12.009.

40. Rezaee N, Barbon C, Zaki A, He J, Salman B, Hruban RH, Cameron JL, Herman JM, Ahuja N, Lennon AM, Weiss MJ, Wood LD, Wolfgang CL. Intraductal papillary mucinous neoplasm (IPMN) with high-grade dysplasia is a risk factor for the subsequent development of pancreatic ductal adenocarcinoma. HPB (Oxford). 2016; 18:236-46. https:// doi.org/10.1016/j.hpb.2015.10.010. [PubMed]

41. Chai SM, Herba K, Kumarasinghe MP, de Boer WB, Amanuel B, Grieu-Iacopetta F, Lim EM, Segarajasingam D, Yusoff I, Choo C, Frost F. Optimizing the multimodal approach to pancreatic cyst fluid diagnosis: developing a volume-based triage protocol. Cancer Cytopathol. 2013; 121:86-100. https://doi.org/10.1002/cncy.21226. [PubMed]
42. Ajaj Saieg M, Munson V, Colletti S, Nassar A. Impact of Pancreatic Cyst Fluid CEA Levels on the Classification of Pancreatic Cysts Using the Papanicolaou Society of Cytology Terminology System for Pancreaticobiliary Cytology. Diagn Cytopathol. 2017; 45:101-06. https://doi. org/10.1002/dc.23633. [PubMed]

43. Zhou AG, Hutchinson LM, Cosar EF. Urine Cytopathology and Ancillary Methods. Surg Pathol Clin. 2014; 7:77-88. https://doi.org/10.1016/j.path.2013.10.003. [PubMed]

44. Pitman MB, Centeno BA, Ali SZ, Genevay M, Stelow E, Mino-Kenudson M, Castillo CF, Schmidt CM, Brugge WR, Layfield LJ. Standardized terminology and nomenclature for pancreatobiliary cytology: The Papanicolaou Society of Cytopathology Guidelines. Cytojournal. 2014; 11:3. https:// doi.org/10.4103/1742-6413.133343. [PubMed]

45. Kamionek M, Ahmadi Moghaddam P, Sakhdari A, Kovach AE, Welch M, Meng X, Dresser K, Tomaszewicz K, Cosar EF, Mark EJ, Fraire AE, Hutchinson L. Mutually exclusive extracellular signal-regulated kinase pathway mutations are present in different stages of multi-focal pulmonary Langerhans cell histiocytosis supporting clonal nature of the disease. Histopathology. 2016; 69:499-509. https://doi. org/10.1111/his.12955. [PubMed]

46. Cornejo KM, Deng A, Wu H, Cosar EF, Khan A, St Cyr M, Tomaszewicz K, Dresser K, O’Donnell P, Hutchinson L. The utility of MYC and FLT4 in the diagnosis and treatment of postradiation atypical vascular lesion and angiosarcoma of the breast. Hum Pathol. 2015; 46:868-75. https://doi. org/10.1016/j.humpath.2015.02.014. [PubMed]

47. Cornejo KM, Hutchinson L, Deng A, Tomaszewicz K, Welch M, Lyle S, Dresser K, Cosar EF. BRAF/KRAS gene sequencing of sebaceous neoplasms after mismatch repair protein analysis. Hum Pathol. 2014; 45:1213-20. https:// doi.org/10.1016/j.humpath.2014.02.001. [PubMed]

48. Liu Q, Tomaszewicz K, Hutchinson L, Hornick JL, Woda B, $\mathrm{Yu} \mathrm{H}$. Somatic mutations in histiocytic sarcoma identified by next generation sequencing. Virchows Arch. 2016; 469:23341. https://doi.org/10.1007/s00428-016-1965-2. [PubMed]

49. Forbes SA, Bindal N, Bamford S, Cole C, Kok CY, Beare D, Jia M, Shepherd R, Leung K, Menzies A, Teague JW, Campbell PJ, Stratton MR, Futreal PA. COSMIC: mining complete cancer genomes in the Catalogue of Somatic Mutations in Cancer. Nucleic Acids Res. 2011; 39:D94550. https://doi.org/10.1093/nar/gkq929. [PubMed]

50. Sherry ST, Ward MH, Kholodov M, Baker J, Phan L, Smigielski EM, Sirotkin K. dbSNP: the NCBI database of genetic variation. Nucleic Acids Res. 2001; 29:308-11. https://doi.org/10.1093/nar/29.1.308. [PubMed]

51. Pan XY, Tsai MH, Wuputra K, Ku CC, Lin WH, Lin YC, Kishikawa S, Noguchi M, Saito S, Lin CS, Yokoyama KK. Application of Cancer Cell Reprogramming Technology to Human Cancer Research. Anticancer Res. 2017; 37:336777. https://doi.org/10.21873/anticanres.11703. [PubMed] 